\title{
Microscopic theory of magnetism in $\operatorname{Sr}_{3} \operatorname{Ir}_{2} \mathrm{O}_{7}$
}

\author{
Jean-Michel Carter ${ }^{1}$ and Hae-Young $\mathrm{Kee}^{1,2, *}$ \\ ${ }^{1}$ Department of Physics, University of Toronto, Toronto, Ontario M5S 1A7 Canada \\ ${ }^{2}$ Canadian Institute for Advanced Research, Toronto, Ontario Canada
}

\begin{abstract}
An intriguing idea of spin-orbit Mott insulator has been proposed to explain magnetic insulating behavior in various iridates. This scenario relies on the strength of the spin-orbit coupling being comparable to electronic correlations, and it is not a priori obvious whether this picture is valid for all iridates. In particular, $\mathrm{Sr}_{3} \operatorname{Ir}_{2} \mathrm{O}_{7}$ exhibits a small charge gap and magnetic moment compared to $\mathrm{Sr}_{2} \mathrm{IrO}_{4}$, questioning the validity of such hypothesis. To understand the microscopic mechanism for magnetism in $\mathrm{Sr}_{3} \mathrm{Ir}_{2} \mathrm{O}_{7}$, we construct a tight binding model taking into account the full $t_{2 g^{-}}$ orbitals, the staggered rotation of the local octahedra, and the bilayer structure. The bands near the Fermi level are mainly characterized by the total angular momentum $J_{\text {eff }}=1 / 2$, except below the $\Gamma$ point, supporting a reasonably strong spin-orbit coupling picture. A first order transition to a collinear antiferromagnet via multi-orbital Hubbard interactions is found within the mean field approximation. The magnetic moment jump at the transition is consistently smaller than $\mathrm{Sr}_{2} \mathrm{IrO}_{4}$, originated from the underlying band structure of a barely band insulator. Given the small charge gap and moment observed in $\mathrm{Sr}_{3} \mathrm{Ir}_{2} \mathrm{O}_{7}$, the system is close to a magnetic transition. A comparison to a spin-model is presented and connection to the Mott insulator is also discussed.
\end{abstract}

\section{INTRODUCTION}

The concept of Mott insulators is prevalent in strongly correlated materials, referring to a strong electronic correlation driven insulator that violates the conventional band theory. Often, Mott insulators are accompanied by antiferromagnetic ordering, but genuine Mott insulators have a robust charge gap above the Néel temperature where the magnetic ordering disappears. Mott insulators have been found in correlated electronic systems with $3 \mathrm{~d}-$ orbital materials including the high transition temperature cuprates, and are considered seeds of exotic phases when doped by holes or electrons. However, with heavier atom systems, the outer shell electron wavefunctions are less localized leading to weaker electronic correlation, and thus band theory should be a good starting point to model the behavior of electrons in such solids.

Surprisingly, $\mathrm{Sr}_{2} \mathrm{IrO}_{4}$, a material with heavy $5 \mathrm{~d}$ Ir atoms, exhibits a magnetic insulating state, despite having partially filled 5d-orbitals. One missing ingredient that is relevant in these heavy elements is spin-orbit coupling (SOC). In particular, when the SOC strength is comparable to that of electronic interactions, understanding their interplay becomes challenging. Iridates with 5 d-orbitals offer such a playground to investigate their combined effects. It has been suggested that due to the narrowing of the bandwidth induced by the strong SOC, the effect of Hubbard interactions is amplified, leading to an insulating state in some layered perovskite ${ }^{1-9}$ and pyrochlore ${ }^{10[11}$ iridates. This state was called a spin-orbit Mott insulator $5[6 \mid 8$ While this proposal of Mott insulator for $\mathrm{Sr}_{2} \mathrm{IrO}_{4}$ itself was challenged in recent studies ${ }^{12 \mid 13}$, the idea has been quickly applied to other iridates including $\mathrm{Sr}_{3} \mathrm{Ir}_{2} \mathrm{O}_{7} \sqrt{14 \mid 15}$, its bilayer sister.

However, optical conductivity $\sqrt{6}$ and transport ${ }^{3[16}$ data have shown that the charge gap in the bilayer system is significantly smaller than in the single layer compound. In addition, the magnetic structures in these two sys- tems differ; $\mathrm{Sr}_{3} \mathrm{Ir}_{2} \mathrm{O}_{7}$ displaying a collinear antiferromagnetic structure with moments aligned with the c-axis $17+21$ whereas $\mathrm{Sr}_{2} \mathrm{IrO}_{4}$ is well-known to have a canted antiferromagnetic structure with moments in the ab-plane ${ }^{5 / 7 / 8}$. This may be related to the crystal structure of the two compounds. $\mathrm{Sr}_{3} \mathrm{Ir}_{2} \mathrm{O}_{7}$ crystallises in a Bbcb space group 22 whereas $\mathrm{Sr}_{2} \mathrm{IrO}_{4}$ does with an $\mathrm{I}_{1} /$ acd space group 1 . Another major difference between these two materials is the giant magnon gap seen in $\mathrm{Sr}_{3} \mathrm{Ir}_{2} \mathrm{O}_{7} \sqrt{14}$ whereas in $\mathrm{Sr}_{2} \mathrm{IrO}_{4}$, the magnon gap, if it exists, is too small for detection with current resonant inelastic x-ray scattering (RIXS) resolution 23 . Based on these differences, it is not clear whether a strong SOC approach is valid $\mathrm{Sr}_{3} \mathrm{Ir}_{2} \mathrm{O}_{7}$.

In this paper, we build a tight-binding Hamiltonian for the bilayer $\mathrm{Sr}_{3} \mathrm{Ir}_{2} \mathrm{O}_{7}$ taking into account the full $t_{2 g}$ manifold, the local staggered rotation of the octahedra, and bilayer coupling, to understand the validity of strong SOC picture and the interplay among SOC, electronic correlation, and the crystal structure. The paper is organized as follows. In Sec. III using a SlaterKoster theory 24 , the tight binding parameters are estimated. Comparing this band structure with recent angle-resolved photoemission spectroscopy (ARPES) $15 \mid 25$ measurements and first-principle calculations 6 , we determined the SOC strength. Indeed the bands near the Fermi level are mainly $J_{\text {eff }}=1 / 2$, except near the $\Gamma$ point below the Fermi level, favoring a reasonably strong SOC limit. Due to the nature of $J_{\text {eff }}=1 / 2$ wavefunction, $\mathrm{Sr}_{3} \mathrm{Ir}_{2} \mathrm{O}_{7}$ bilayer material exhibits a nearly band insulator distinctly different from $\mathrm{Sr}_{2} \mathrm{IrO}_{4}$. This originates from large bilayer hopping terms and the alternating rotations of the local octahedra. We then study the effects of electronic interactions using a multi-orbital Hubbard model with Hund's coupling in Sec. III. A first order transition is found within mean field theory, and the jump in the magnetization and the critical interaction strength depend on the SOC value. The implications of our study 
in the context of recent experimental results are discussed in the section IV.

\section{TIGHT-BINDING MODEL}
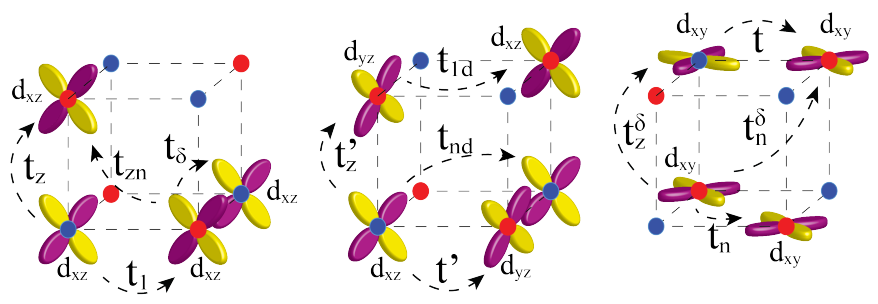

FIG. 1. [color online] Tight binding hopping parameters: the red and blue dots represent two different Ir atom environments due to staggered octahedra rotation. $\left(t, t_{1}, t_{z}, t_{\delta}, t_{z}^{\delta}\right)$ are NN hopping between the same orbitals, while $\left(t^{\prime}, t_{z}^{\prime}\right)$ between different orbitals. $\left(t_{n}, t_{z n}, t_{n d}, t_{n}^{\delta}\right) /\left(t_{1 d}\right)$ are next NN hopping integrals between the same/different orbitals.

The bilayer structure is represented by a dashed cube in Fig. 1, with the red and blue vertices denoting the two different Ir atoms in the bilayer structure due to $\mathrm{IrO}_{6}$ staggered rotation ${ }^{26}$ (the in-plane unit cell area is thus doubled). Taking into account the $t_{2 g}$ d-orbital $\alpha=y z, x z, x y$ with momentum $\mathbf{k}$, sublattice $\gamma=$ blue(B), $\operatorname{red}(\mathrm{R})$, and spin $\sigma$, the tight binding Hamiltonian can be written as $\sum_{k} \psi_{k, l}^{\dagger} H_{0}^{l l^{\prime}} \psi_{k, l^{\prime}}$, with spinor $\psi_{k, l}=\left(d_{k, \downarrow}^{l, B, y z}, d_{k, \downarrow}^{l, B, x z}, d_{k, \uparrow}^{l, B, x y}, B \Leftrightarrow R, \uparrow \Leftrightarrow \downarrow\right)^{T}$ where $d_{k, \sigma}^{l, \gamma, \alpha}$ is a annihilation operator at the layer $l, l^{\prime}=1,2$, sublattice $\gamma$ and orbital $\alpha$. Using this basis, the matrices $H_{0}^{l l^{\prime}}$ including nearest neighbor $(\mathrm{NN})$ and next $\mathrm{NN}$ hoppings, is given by

$H_{0}^{l l^{\prime}}=\left(\begin{array}{cc}H_{s o} \delta_{l l^{\prime}}+H_{B B}^{l l^{\prime}} & H_{B R}^{l l^{\prime}} \\ H_{B R}^{l l^{\prime} \dagger} & H_{s o} \delta_{l l^{\prime}}+H_{R R}^{l l^{\prime}}\end{array}\right)+[$ time-reversed]

with $H_{\text {so }}$ being the atomic spin-orbit coupling, $\lambda \mathbf{L}_{i} \cdot \mathbf{S}_{i}$. The intra-layer intra-sublattice hopping (next $\mathrm{NN}$ ) of $H_{B B}^{l l}=H_{R R}^{l l}$ are given by

$$
H_{s o}+H_{B B}^{l l}=\left(\begin{array}{ccc}
\epsilon^{y z} & -i \frac{\lambda}{2}+\epsilon_{1 d} & \frac{\lambda}{2} \\
i \frac{\lambda}{2}+\epsilon_{1 d} & \epsilon^{x z} & i \frac{\lambda}{2} \\
\frac{\lambda}{2} & -i \frac{\lambda}{2} & \epsilon^{x y}
\end{array}\right)
$$

where $\epsilon^{x y}=4 t_{n} \cos \left(k_{x}\right) \cos \left(k_{y}\right)+\mu_{x y}, \epsilon^{y z}=\epsilon^{x z}=$ $4 t_{n d} \cos k_{x} \cos k_{y}$, and $\epsilon_{1 d}=4 t_{1 d} \sin k_{x} \sin k_{y}$. $\mu_{x y}$ denotes the atomic potential difference between $x y$ and onedimensional $x z / y z$ orbitals due to tetragonal distortion. $H_{B R}^{l l}$ is NN hopping terms between B and R sublattice sites, and is written as

$$
H_{B R}^{l l}=\left(\begin{array}{ccc}
\epsilon_{k}^{y z} & \epsilon_{k}^{r o t} & 0 \\
-\epsilon_{k}^{r o t} & \epsilon_{k}^{x z} & 0 \\
0 & 0 & \epsilon_{k}^{x y}
\end{array}\right)
$$

where the dispersions are given by $\epsilon_{k}^{y z}=2\left(t_{1} \cos \left(k_{y}\right)+\right.$ $\left.t^{\delta} \cos \left(k_{x}\right)\right), \quad \epsilon_{k}^{x z}=2\left(t_{1} \cos \left(k_{x}\right)+t^{\delta} \cos \left(k_{y}\right)\right), \epsilon_{k}^{x y}=$ $2 t\left(\cos \left(k_{x}\right)+\cos \left(k_{y}\right)\right)$, and $\epsilon_{k}^{r o t}=2 t^{\prime}\left(\cos \left(k_{x}\right)+\cos \left(k_{y}\right)\right)$. For the bilayer hopping terms, it is important to notice that a $\mathrm{R}(\mathrm{B})$ atom on one layer lies on top of a $\mathrm{B}(\mathrm{R})$ on the other layer. Therefore, the bilayer hopping terms in $H_{0}^{12}$ are given by

$$
H_{B R}^{12}=\left(\begin{array}{ccc}
t_{z} & t_{z}^{\prime} & 0 \\
-t_{z}^{\prime} & t_{z} & 0 \\
0 & 0 & t_{z}^{\delta}
\end{array}\right) ; H_{B B}^{12}=H_{R R}^{12}=\left(\begin{array}{ccc}
\epsilon_{d}^{y z} & 0 & 0 \\
0 & \epsilon_{d}^{x z} & 0 \\
0 & 0 & \epsilon_{d}^{x y}
\end{array}\right),
$$

where $\epsilon_{d}^{y z}=2 t_{z n} \cos k_{y}, \epsilon_{d}^{x z}=2 t_{z n} \cos k_{x}$, and $\epsilon_{d}^{x y}=$ $4 t_{n}^{\delta} \cos k_{x} \cos k_{y}$. The hopping parameters of $\left(t, t_{1}, t_{z}, t^{\prime}\right.$, $\left.t_{z}^{\prime}, t_{n}, t_{z n}, t_{1 d}, t^{\delta}, t_{z}^{\delta}, t_{n d}, t_{n}^{\delta}\right)$ are shown in Fig. 1 .

Setting $t$ as a unit, there appears to be 11 other hopping parameters, but they are not all independent of each other. Note that in an ideal octahedra, $\mu_{x y}=0$, $t=t_{1}=t_{z}, t_{n}=t_{z n}$, and $t^{\delta}=t_{z}^{\delta}$ based on cubic symmetry, while $t^{\prime}=t_{z}^{\prime}=0$ without the staggered rotation of octahedra. Due to the $\mathrm{IrO}_{6}$ rotation, the above relations break down and how they differ depends on the angle of the staggered rotation. Using Slater-Koster theory 24 with $t_{d d \sigma}: t_{d d \pi}: t_{d d \delta}=3 / 2:-1: 1 / 4^{27}$, and taking into account a distance factor of 0.9 for bilayer terms and of 0.2 for the next $\mathrm{NN}$ for the exponential suppression of hopping parameters with distance, we found $\left(t, t_{1}, t_{z}, t^{\prime}\right.$, $\left.t_{z}^{\prime}, t_{n}, t_{z n}, t_{1 d}, t^{\delta}, t_{z}^{\delta}, t_{n d}, t_{n}^{\delta}\right)=(-1.0,-0.94,-0.8,0.15$, $0.36,0.16,0.2,0.11,0.27,0.15,0,0)$ for a staggered rotation angle of $\pm 12^{\circ}$. Once the ratio between $t_{d d \sigma}, t_{d d \pi}$, and $t_{d d \delta}$ is fixed, only the SOC $\lambda$ and the tetragonal splitting $\mu_{x y}$ remain independent parameters.

The tight binding band structure is shown in Fig. 2 for $\lambda / t=3$ and $\mu_{x y}=0$, where $J_{\text {eff }}=1 / 2$ bands are in red, while $J_{\text {eff }}=3 / 2$ bands are in blue. There are several important features to notice. First, $J_{\text {eff }}=1 / 2$ and $3 / 2$ bands below the Fermi level are mixed along $\Gamma$ to $M=(\pi / 2, \pi / 2)$ and along $\Gamma$ to $X=(\pi, 0)$, and the bands near $\Gamma$ are $J_{\text {eff }}=3 / 2$ states, not $1 / 2$. Second, this band structure is similar to recently reported ARPES data ${ }^{15 \mid 25}$ on $\mathrm{Sr}_{3} \mathrm{Ir}_{2} \mathrm{O}_{7}$ and a first principle calculation ${ }^{6}$, but the bands near $\Gamma$ have been misidentified as $J_{\text {eff }}=1 / 2$ in these works 6 Third, our results imply that the SOC is large, but not enough to fully separate $J_{\text {eff }}=1 / 2$ and $3 / 2$ bands below the Fermi level, similar to theoretical studies on $\mathrm{Sr}_{2} \mathrm{IrO}_{4}$ 1228. On the other hand, the unoccupied bands above the chemical potential are pure $J_{\text {eff }}=1 / 2$. Thus the RIXS intensity contains more $J_{\text {eff }}=1 / 2$ band contribution, because it is from a combination of unoccupied and occupied bands, while ARPES measures only occupied states.

The $M-X$ path is the reduced Brillouin zone (BZ) boundary due to the staggered octahedra rotation, and the degeneracy is protected because the in-plane potential generated by the staggered rotation has the form of $\cos k_{x}+\cos k_{y}$ which is absent along this path. The energy difference in $J_{\text {eff }}=1 / 2$ bands is largest at $X$ point, because of a constructive combination of $t, t_{z}, t^{\prime}$, and 

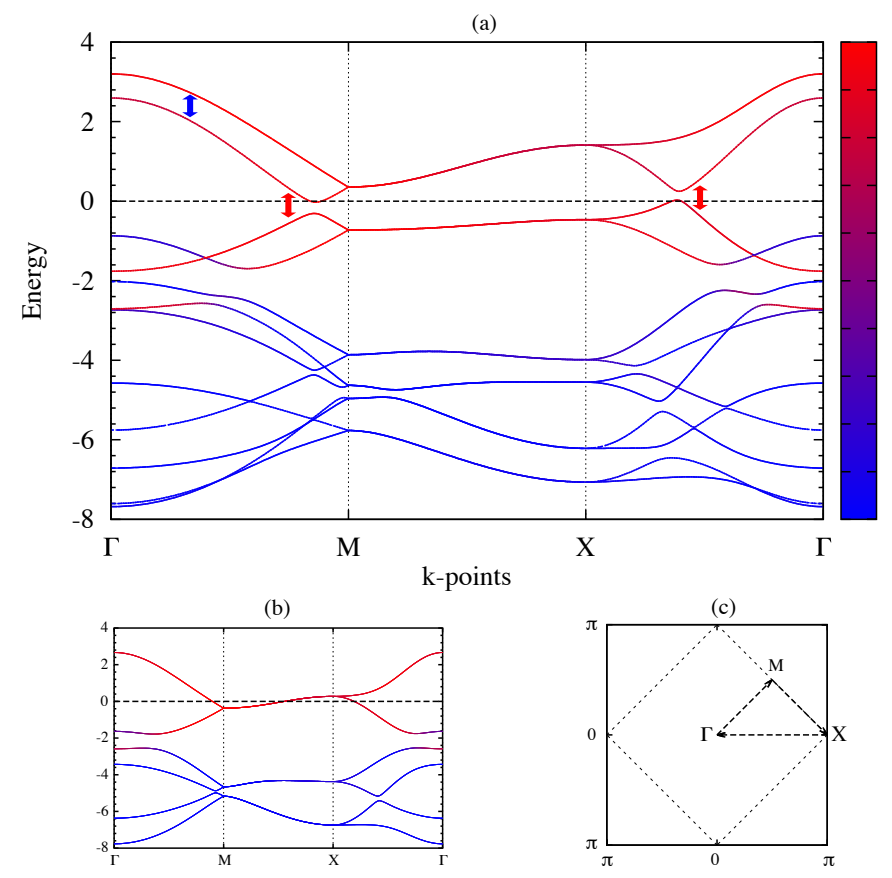

FIG. 2. [color online] (a) Underlying band structure of $\mathrm{Sr}_{3} \mathrm{Ir}_{2} \mathrm{O}_{7}$ along the path shown in (c). The weight of the $J_{\text {eff }}=1 / 2$ state is colored in red while the $J_{\text {eff }}=3 / 2$ weight is in blue, and we see that the relevant bands near the Fermi level is mostly composed of $J_{\text {eff }}=1 / 2$ wavefunction, but note that the band immediately below the Fermi level around the $\Gamma$ point is made of $J_{\text {eff }}=3 / 2$ state. The band structure is distinct from the single layered $\mathrm{Sr}_{2} \mathrm{IrO}_{4}$ system shown in (b) mainly due to the large bilayer hoppings inherited from the nature of the $J_{\text {eff }}=1 / 2$ wavefunction.

$t_{z}^{\prime}$. A typical splitting of $0.8 t$ could be measured by the separation of the two unoccupied bands of $J_{\text {eff }}=1 / 2$ near the $\Gamma$ point (denoted by the blue arrow in Fig. 2 a). In Ref. 15, the bilayer splitting was estimated by the separation between the two highest occupied bands at $\Gamma$. However, the energy difference between either the 1st and 2nd or the 1st and 3rd highest occupied band at the $\Gamma$ point in Fig. 2(a) is due to both the bilayer hoppings and the hybridization between $J_{\text {eff }}=1 / 2$ and $3 / 2$ bands. This splitting further gives an estimate of our unit $t \sim 200 \mathrm{meV}$, which is consistent with the overall bandwidth when comparing our tight binding spectrum with the ARPES data15.

Last, the most important result is the topology of the band structure. It is almost a band insulator where the Fermi level barely touches the bottom and top of unoccupied and occupied bands near $M$ and $X$ respectively. A small difference in the hopping parameters could change particular features such as the size of the small hole and electron pockets, but the separation of the two unoccupied bands from the rest via a direct gap at every $k$-point, does not depend on the details. The direct gap between these two unoccupied bands occurs due to a finite $t^{\prime}$ or $t_{z}^{\prime}$, shown as the red arrow in Fig. 2(a), indicating the importance of staggered octahedra rotation between the NN Ir atoms. For comparison, we present the underlying band structure of the single layer iridates in Fig. 2(b) that shows the large Fermi surface crossing along the M$\mathrm{X}$ path. Now that we are equipped with the proper tight binding model, let us move to a magnetic ordering mechanism.

\section{MEAN-FIELD THEORY}

A general interaction Hamiltonian in multi-orbital systems is given by

$H_{i n t}=U \sum_{i} n_{i \alpha \uparrow} n_{i \alpha \downarrow}+U^{\prime} \sum_{i, \alpha \neq \beta} n_{i \alpha} n_{i \beta}-J \sum_{i \alpha \neq \beta} \mathbf{S}_{i \alpha} \cdot \mathbf{S}_{i \beta}$,

where the Hund's coupling $J=\left(U-U^{\prime}\right) / 2$ is determined by intra- and inter-orbital Hubbard $U$ and $U^{\prime}$ and where we set $U^{\prime}=0.8 U^{\sqrt{12 \mid 29}}$. We treat these interactions at the mean-field (MF) level in the magnetic channels to find possible magnetic orderings. To consider the strong SOC, we define the order parameter in the $J_{\text {eff-basis. To }}$ do so, we first rewrite the above $H_{\text {int }}$ in the $J_{\text {eff-basis, }}$ and decouple all the terms in the magnetic channels. The mean field Hamiltonian is then given by $H_{i n t}^{M F}=$ $-\left(\frac{U}{3}+\frac{2 U^{\prime}}{3}-\frac{2 J}{3}\right) \mathbf{m}_{i 1} \cdot \mathbf{S}_{i 1}-\left(\frac{U}{2}+\frac{U^{\prime}}{2}-\frac{J}{2}\right) \sum_{n=2,3} \mathbf{m}_{i n}$. $\mathbf{S}_{i n}$. Here $\mathbf{m}_{i n}=\left\langle\mathbf{S}_{i n}\right\rangle=\frac{1}{2}\left\langle c_{i n \mu}^{\dagger} \vec{\sigma}_{\mu \nu} c_{i n \nu}\right\rangle$, where $\vec{\sigma}$ is the Pauli matrix for pseudospin for $n=1,2,3$ that correspond to $\left(J_{\text {eff }}, J_{\text {eff }}^{z}\right)=(1 / 2, \pm 1 / 2),(3 / 2, \pm 3 / 2)$, and $(3 / 2, \pm 1 / 2)$, respectively. The order parameters $m_{i, n}$ are then determined self-consistently. There are $36 \mathrm{MF}$ order parameters from three pseudospin states $(n)$, two sublattices $(\gamma)$, two layers $(l)$, and three directions of $\mathbf{m}$.

As $U$ increases, there is a weakly first order phase transition from a barely band insulator into a magnetically ordered phase as shown in Fig. 3. $\mathbf{m}_{1}$ is finite and the magnetic pattern is given by a G-type antiferromagnetic order where the moments in the blue and red atoms point in opposite directions. The $\mathbf{m}_{2}$ and $\mathbf{m}_{3}$ order parameters are smaller by two orders of magnitude, confirming that the moments are made of $J_{\text {eff }}=1 / 2$ electrons. The critical interaction $U_{c}$ depends on the SOC strength. The bigger the SOC, the smaller the $U_{c}$ is, similar to the single layer case reported in Ref. 28 and confirmed here, but opposite to three dimensional materials, $\mathrm{SrIrO}_{3}{ }_{30}^{30}$.

A finite moment affects the band structure, where the bottom of electron and top of hole bands are pushed away from the Fermi level as shown in Fig. 4, making a charge gap visible. For comparison, we show the band structure after magnetic ordering for the $\mathrm{Sr}_{2} \mathrm{IrO}_{4}$ system also, where the bands are much more affected by the ordering than in the $\mathrm{Sr}_{3} \mathrm{Ir}_{2} \mathrm{O}_{7}$ system. When $U>U_{c} \sim 3 t$ for $\lambda / t=3$, there are two competing ordering patterns - canted antiferromagnet (AF) and collinear AF. We found that when the ratio of $t_{z}^{\prime} / t_{z}$ is equal to the ratio of $t^{\prime} / t$, the canted $\mathrm{AF}$ and collinear AF become degenerate. 


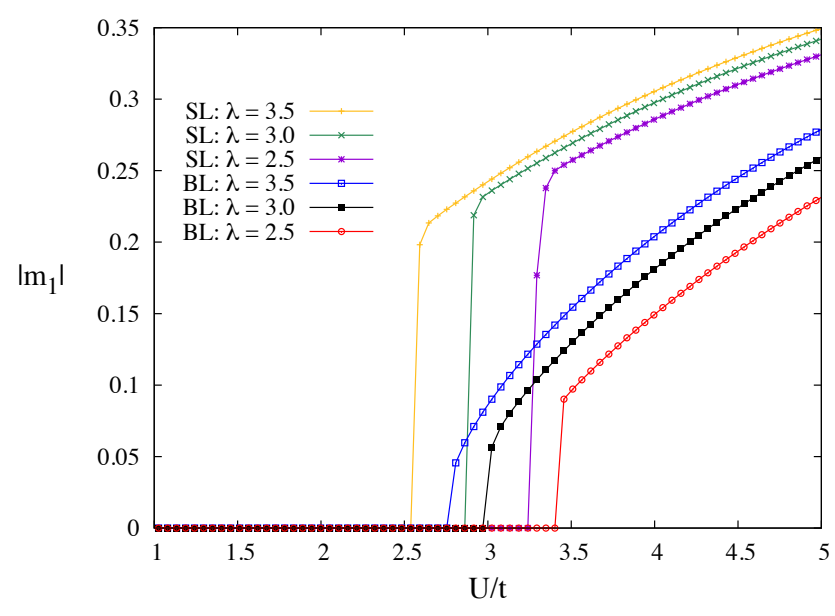

FIG. 3. [color online] First order phase transition is found for bilayer (BL) $\mathrm{Sr}_{3} \mathrm{Ir}_{2} \mathrm{O}_{7}$ for $\lambda / t=2.5,3$ and 3.5. For comparison, the transition for single layer (SL) $\mathrm{Sr}_{2} \mathrm{IrO}_{4}$ is also shown for the same SOC strengths. We note that for the same set of parameters, the $\mathrm{Sr}_{3} \mathrm{Ir}_{2} \mathrm{O}_{7}$ requires a higher $U_{c}$ for the magnetic moment to set in, due to its underlying band insulator. Note that only $\left|\mathbf{m}_{1}\right|$ is shown in the figure since $\left|\mathbf{m}_{2}\right|$ and $\left|\mathbf{m}_{3}\right|$ are negligible.

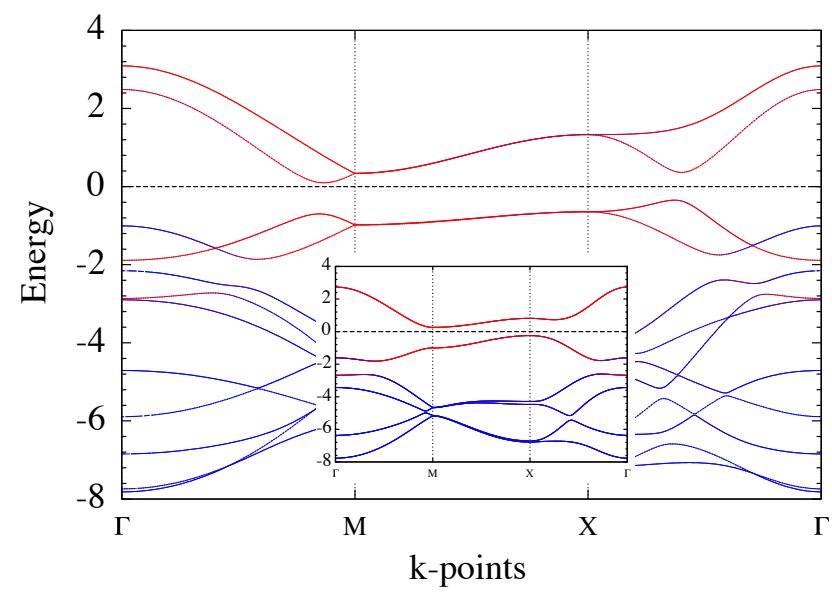

FIG. 4. [color online] Band structure with magnetic ordering for $\mathrm{Sr}_{3} \mathrm{Ir}_{2} \mathrm{O}_{7}$ (and $\mathrm{Sr}_{2} \mathrm{IrO}_{4}$ in inset) using the self-consistent solution at $U / t=4$. The charge gap from these calculation are $\Delta_{c} \sim 0.4 t$ for $\mathrm{Sr}_{3} \mathrm{Ir}_{2} \mathrm{O}_{7}$ and $\Delta_{c} \sim 0.6 t$ for $\mathrm{Sr}_{2} \mathrm{IrO}_{4}$.

From the Slater-Koster theory shown above, $t_{z}^{\prime} / t_{z} \approx 2.4$ $t^{\prime} / t$, and a G-type collinear AF state along the c-axis is favored by the bilayer coupling. Such a state has been recently confirmed in experiments 17,21 .

Given the small magnetic moment observed in $\mathrm{Sr}_{3} \mathrm{Ir}_{2} \mathrm{O}_{7} \frac{9}{}$, the system is close to a magnetic transition, where the size of the charge gap from transport data does not support a strongly correlated insulator. ${ }^{3}$ Indeed when the magnetic moment disappears, the band structure is almost a band insulator where the Fermi level barely touches the bottom/top of electron/hole bands. However, the magnetic moment appears only in $J_{\text {eff }}=1 / 2$ channel, and one may ask if this magnetic insulator is connected to the large $U$-limit of spin-orbit Mott insulator. The spin-model derived from a $J_{\text {eff }}=1 / 2$ only mode ${ }^{31}$ indeed displays the same magnetic order, suggesting that the two limits are adiabatically connected.

\section{DISCUSSION AND SUMMARY}

Recently, various measurements on $\mathrm{Sr}_{3} \mathrm{Ir}_{2} \mathrm{O}_{7}$ using different techniques, such as ARPES 15|25], neutron scattering 19 , and RIX $S^{9 / 14} 32$, in addition to transport $3 \sqrt{36}$ and optical studies 6 , have been reported. It is important to ask whether the current microscopic theory is compatible with the measured quantities, and further, if it offers a useful starting point for future studies.

First of all, the latest ARPES data ${ }^{15 \mid 25}$ provide the dispersion of the occupied bands. Using Slater-Koster hopping integrals for d-orbitals and considering the local distortion of the octahedra as well as a distance factor that is exponentially decaying with distance, we obtained the hopping parameters up to next-nearest-neighbor. Note that the only independent parameters left were the SOC strength and the tetragonal distortion. Comparing the computed band structures and ARPES data, we show that the band below the Fermi energy is indeed mainly $J_{\text {eff }}=1 / 2$, except near the $\Gamma$ point where there is a large contribution from $J_{\text {eff }}=3 / 2$. Overall, our tight-binding band structure fits well with first principle calculations 6 and the recent ARPES data ${ }^{15 / 25}$.

Furthermore, the current mean field study shows that the jump in the magnetic moment at the first order transition is weaker in the bilayer iridates than the single layer iridates. This is due to the band structure being nearly insulating, advancing our understanding of the smaller magnetic moment in bilayer iridates 9 . As a consequence, the size of the charge gap is smaller in the bilayer system compared with the single layer one, agreeing with transport ${ }^{3 / 16}$ and optical conductivity ${ }^{6}$ data.

A remaining puzzle is the giant magnon gap of over $90 \mathrm{meV}$ reported in the RIXS data 14 . Our microscopic model contains terms that break the spin rotation symmetry, a source for the magnon gap. Using a second order perturbation theory, this microscopic model yields a spin-wave spectrum with a magnon gap of approximately $35 \mathrm{meV}$, assuming that $t=200 \mathrm{meV}$ and $U / t=4$. The gap originates from the c-axis anisotropic exchange terms being different from the in-plane ones. Since the tight binding parameters fit the ARPES data well, this discrepancy in the size of the magnon gap implies that the second order perturbation assuming a large $U / t$ limit is not appropriate to estimate the magnon gap reported in the RIXS spectrum, and one should add higher order terms in the perturbation theory. In this intermediate- $U$ range, the spin susceptibility using the random phase approximation might be a more appropriate approach than the semi-classical spin wave theory applied to the spin model obtained in the large- $U$ limit. Our microscopic 
model is a useful starting point for future study of the spin susceptibility for general wavevector and frequency.

In summary, we build a tight binding model for $\mathrm{Sr}_{3} \mathrm{Ir}_{2} \mathrm{O}_{7}$ and show that the non-interacting system is almost a band insulator where the two unoccupied $J_{\text {eff }}=$ $1 / 2$ bands are separated from the rest of occupied bands by a direct gap at every k-point. As the interaction strength increases, a band insulator to magnetic insulator transition occurs, and a finite magnetic moment pushes the top/bottom of hole/electron bands further away from the Fermi level making the direct band gap bigger. This is qualitatively different from the single layer $\mathrm{Sr}_{2} \mathrm{IrO}_{4}$, where the degeneracy of the bands crossing the Fermi level along $M-X$ is lifted turning it into a magnetic insulator 28 The ground state magnetic ordering pattern is sensitive to the lattice structure, with the staggered rotation of $\mathrm{IrO}_{6}$ octahedra between adjacent layers playing a crucial role in both developing a band insulator in the tight binding spectrum and determining the canted AF ordering pattern. $\mathrm{Sr}_{3} \mathrm{Ir}_{2} \mathrm{O}_{7}$ is a magnetic insulator with a small moment ${ }^{9}$, and thus it is close to the transition. However, the AF ordering pattern obtained from the spin model derived in the large $U$-limit $\frac{31}{31}$ is identical to that obtained from the current mean-field theory, implying that this small moment insulator is likely smoothly connected to the Mott insulating regime as $U$ increases, where the charge gap persists above the magnetic ordering temperature.

Acknowledgement - This work was supported by NSERC of Canada.
* hykee@physics.utoronto.ca

1 M. K. Crawford, M. A. Subramanian, R. L. Harlow, J. A. Fernandez-Baca, Z. R. Wang, and D. C. Johnston, Phys. Rev. B 49, 9198 (1994).

${ }^{2}$ G. Cao, J. Bolivar, S. McCall, J. E. Crow, and R. P. Guertin, Phys. Rev. B 57, R11039 (1998)

3 G. Cao, Y. Xin, C. S. Alexander, J. E. Crow, P. Schlottmann, M. K. Crawford, R. L. Harlow, and W. Marshall, Phys. Rev. B 66, 214412 (2002)

4 I. Nagai, Y. Yoshida, S. I. Ikeda, H. Matsuhata, H. Kito, and M. Kosaka, Journal of Physics: Condensed Matter 19, 136214 (2007)

${ }^{5}$ B. J. Kim, H. Jin, S. J. Moon, J.-Y. Kim, B.-G. Park, C. S. Leem, J. Yu, T. W. Noh, C. Kim, S.-J. Oh, J.-H. Park, V. Durairaj, G. Cao, and E. Rotenberg, Phys. Rev. Lett. 101, 076402 (2008)

${ }^{6}$ S. J. Moon, H. Jin, K. W. Kim, W. S. Choi, Y. S. Lee, J. Yu, G. Cao, A. Sumi, H. Funakubo, C. Bernhard, and T. W. Noh, Phys. Rev. Lett. 101, 226402 (2008)

7 G. Jackeli and G. Khaliullin, Phys. Rev. Lett. 102, 017205 (2009)

$\checkmark$ B. J. Kim, H. Ohsumi, T. Komesu, S. Sakai, T. Morita, H. Takagi, and T. Arima, Science 323, 1329 (2009).

9 S. Fujiyama, K. Ohashi, H. Ohsumi, K. Sugimoto, T. Takayama, T. Komesu, M. Takata, T. Arima, and H. Takagi, ArXiv e-prints (2012), arXiv:1207.7151 [condmat.str-el]

${ }^{10}$ D. Pesin and L. Balents, Nat Phys 6, 376 (2010).

11 B.-J. Yang and Y. B. Kim, Phys. Rev. B 82, 085111 (2010)

12 R. Arita, J. Kuneš, A. V. Kozhevnikov, A. G. Eguiluz, and M. Imada, Phys. Rev. Lett. 108, 086403 (2012).

13 D. Hsieh, F. Mahmood, D. H. Torchinsky, G. Cao, and N. Gedik, Phys. Rev. B 86, 035128 (2012)

14 J. Kim, A. H. Said, D. Casa, M. H. Upton, T. Gog, M. Daghofer, G. Jackeli, J. van den Brink, G. Khaliullin, and B. J. Kim, ArXiv e-prints (2012), arXiv:1205.5337 [cond-mat.str-el]

is Q. Wang, Y. Cao, J. A. Waugh, S. R. Park, T. F. Qi, O. B. Korneta, G. Cao, and D. S. Dessau, ArXiv e-prints (2012), arXiv:1210.4141 [cond-mat.str-el].

16 M. Ge, T. F. Qi, O. B. Korneta, D. E. De Long, P. Schlottmann, W. P. Crummett, and G. Cao, Phys. Rev. B 84, 100402 (2011).
17 J. W. Kim, Y. Choi, J. Kim, J. F. Mitchell, G. Jackeli, M. Daghofer, J. van den Brink, G. Khaliullin, and B. J. Kim, ArXiv e-prints (2012), arXiv:1205.4381 [condmat.str-el].

18 J. Kim, A. H. Said, D. Casa, M. H. Upton, T. Gog, M. Daghofer, G. Jackeli, J. van den Brink, G. Khaliullin, and B. J. Kim, ArXiv e-prints (2012), arXiv:1205.5337 [cond-mat.str-el].

${ }^{19}$ C. Dhital, S. Khadka, Z. Yamani, C. de la Cruz, T. C. Hogan, S. M. Disseler, M. Pokharel, K. C. Lukas, W. Tian, C. P. Opeil, Z. Wang, and S. D. Wilson, Phys. Rev. B 86, 100401 (2012)

${ }^{20}$ S. Boseggia, R. Springell, H. Walker, A. Boothroyd, D. Prabhakaran, S. Collins, and D. McMorrow, ArXiv e-prints (2012), arXiv:1207.0173 [cond-mat.str-el]

21 J. P. Clancy, K. W. Plumb, C. S. Nelson, Z. Islam, G. Cao, T. Qi, and Y.-J. Kim, ArXiv e-prints (2012), arXiv:1207.0960 [cond-mat.str-el]

22 H. Matsuhata, I. Nagai, Y. Yoshida, S. Hara, S. ichi Ikeda, and N. Shirakawa, Journal of Solid State Chemistry 177, 3776 (2004).

${ }^{23}$ J. Kim, D. Casa, M. H. Upton, T. Gog, Y.-J. Kim, J. F. Mitchell, M. van Veenendaal, M. Daghofer, J. van den Brink, G. Khaliullin, and B. J. Kim, Phys. Rev. Lett. 108, 177003 (2012)

24 J. C. Slater and G. F. Koster, Phys. Rev. 94, 1498 (1954).

25 B. M. Wojek, M. H. Berntsen, S. Boseggia, A. T. Boothroyd, D. Prabhakaran, D. F. McMorrow, H. M. Rønnow, J. Chang, and O. Tjernberg, Journal of Physics: Condensed Matter 24, 415602 (2012)

20 M. A. Subramanian, M. K. Crawford, and R. L. Harlow, Materials Research Bulletin 29, 645 (1994).

27 O. Andersen, Solid State Communications 13, 133 (1973).

${ }^{28}$ H. Watanabe, T. Shirakawa, and S. Yunoki, Phys. Rev. Lett. 105, 216410 (2010).

${ }^{29}$ T. Mizokawa and A. Fujimori, Phys. Rev. B 51, 12880 (1995)

so M. Ahsan Zeb and H.-Y. Kee, ArXiv e-prints (2012), arXiv:1206.5836 [cond-mat.str-el].

31 J.-M. Carter and H.-Y. Kee, ArXiv e-prints (2012), arXiv:1207.2183 [cond-mat.str-el].

32 J. W. Kim, Y. Choi, J. Kim, J. F. Mitchell, G. Jackeli, M. Daghofer, J. van den Brink, G. Khaliullin, and 
B. J. Kim, ArXiv e-prints (2012), arXiv:1205.4381 [condmat.str-el] 